American Journal of Biochemistry and Biotechnology 4 (2): 218-225, 2008

ISSN 1553-3468

(C) 2008 Science Publications

\title{
Evidence of Oxidative Stress in Autism Derived from Animal Models
}

\author{
${ }^{1}$ Xue Ming, ${ }^{2}$ Michelle A. Cheh and ${ }^{2}$ Carrie L. Yochum, \\ ${ }^{3}$ Alycia K. Halladay and ${ }^{2}$ George C. Wagner \\ ${ }^{1}$ Pediatric Neuroscience, UMDNJ, Newark, NJ \\ ${ }^{2}$ Psychology, Rutgers University, New Brunswick, NJ \\ ${ }^{3}$ Autism Speaks, Princeton, NJ
}

\begin{abstract}
Autism is a pervasive neurodevelopmental disorder that leads to deficits in social interaction, communication and restricted, repetitive motor movements. Autism is a highly heritable disorder, however, there is mounting evidence to suggest that toxicant-induced oxidative stress may play a role. The focus of this article will be to review our animal model of autism and discuss our evidence that oxidative stress may be a common underlying mechanism of neurodevelopmental damage. We have shown that mice exposed to either methylmercury (MeHg) or valproic acid (VPA) in early postnatal life display aberrant social, cognitive and motor behavior. Interestingly, early exposure to both compounds has been clinically implicated in the development of autism. We recently found that Trolox, a water-soluble vitamin E derivative, is capable of attenuating a number of neurobehavioral alterations observed in mice postnatally exposed to $\mathrm{MeHg}$. In addition, a number of other investigators have shown that oxidative stress plays a role in neural injury following $\mathrm{MeHg}$ exposure both in vitro and in vivo. New data presented here will show that VPA-induced neurobehavioral deficits are attenuated by vitamin $\mathrm{E}$ as well and that the level of glial fibrillary acidic protein (GFAP), a marker of astrocytic neural injury, is altered following VPA exposure. Collectively, these data indicate that vitamin $\mathrm{E}$ and its derivative are capable of protecting against neurobehavioral deficits induced by both $\mathrm{MeHg}$ and VPA. This antioxidant protection suggests that oxidative stress may be a common mechanism of injury leading to aberrant behavior in both our animal model as well as in the human disease state.
\end{abstract}

Key words: Vitamin E, trolox, valproic acid

\section{INTRODUCTION}

The core symptoms of autism include language deficits, impaired social interactions and inappropriate, stereotypic and sometimes self-injurious behaviors. The etiology of autism remains unknown but may involve early exposure to environmental toxicants acting upon genetically-sensitive individuals. No single toxicant has been identified; rather a broad range of toxicants including drugs, metals, solvents, herbicides, pesticides, etc. have been associated with autism ${ }^{[1-3]}$. A common feature across this range of potential compounds is toxicant-induced oxidative stress causing neuronal damage leading to the behavioral phenotype of autism $^{[4-6]}$. Likewise, no single gene has been identified but, rather, a constellation of as many as 15 polymorphisms may ultimately predispose the individual to autism. Again, genetic alterations leading to compromised handling of toxicant-induced reactive oxygen species (ROS) has been a common theme.
Since the etiology of autism is unknown, it is essential that animal models be developed. The behavioral symptoms of autism have proven difficult to model in other species. Accordingly, we have initiated work on a novel strategy to model the behavioral phenotype of autism in mice ${ }^{[1]}$. In this model, the normal development of key behaviors is carefully monitored from birth through adolescence. Once the maturation of these key behaviors is understood in terms of the postnatal day(s) of life in which subjects are able to successfully perform the task or engage in the behavior, the performance of mice with early toxicant exposure and/or genetic modification can be assessed.

The model strategy begins by characterizing behavioral manifestations of developmental disorders as retardations (a behavior fails to develop during a critical period of maturation), regressions (a behavior develops at about the right time but then is lost with

Corresponding Author: George C. Wagner, Psychology, Busch Campus, Rutgers University, New Brunswick, NJ 08854 Tel: 732-445-4660 Fax: 732-445-2263 
later development, especially following toxicant exposure), or intrusions (the appearance of behaviors aberrant in form or frequency which mask normal development). Most developmental disorders include some combination of these conditions. In this framework, the hypothesis that environmental toxicants or genetic alterations are causally involved in autism can be readily tested. That is, acute or repeated exposure to a toxicant should disrupt neurobehavioral development causing behavioral retardation, regression, or intrusions and these toxicant-induced behavioral deficits should occur at lower doses in the geneticallysensitive mice. Traditionally, animal models of developmental disorders have not examined these three scenarios of retardations, regressions and/or intrusions but, instead, focus on single aspects of neurobehavioral development. The judicious use of toxicants associated with autism or toxicants known to damage brain regions associated with autism confers some selectivity of the model for autism. Likewise, manipulation of genes associated with autism also confers some selectivity of this model for autism. Finally, administering a battery of tests that assess social, cognitive and motor maturation of the mice confers some selectivity for autism. Ultimately, it is the possibility of combining select toxicant exposure in genetically-sensitive mice followed by thorough assessment of social, cognitive and motor skill maturation that makes this a comprehensive animal model of autism.

In our initial studies, we identified toxicant induced retardation of motor and cognitive skills following preor post-natal exposure to sodium valproate (VPA). Likewise, we were able to demonstrate dramatic loss of acquired skills, i.e. regressions, following post-natal VPA administration ${ }^{[1]}$. Finally, we demonstrated toxicant induced intrusions wherein toxicant-treated subjects exhibited dramatic increases in stereotypic and self-injurious behaviors akin to those seen in autism ${ }^{[7,8]}$.

VPA was chosen as our first agent to test this model following reports of an association between autism and prenatal exposure to this teratogen ${ }^{[9-13]}$. Previous studies have also demonstrated impairment in cognitive, motor, attention and social development in rats administered pre- or post-natal $\mathrm{VPA}^{[14-17]}$. Accordingly, in our first studies ${ }^{[1]}$ mice were exposed to VPA either in utero or post-natally. The prenatal exposure time reflected a period of cerebellar Purkinje cell generation differentiation in the mouse ${ }^{[10,14,17,18]}$. The post-natal time of P14 was based on our observation that critical cerebellar-mediated behaviors of mid-air righting and negative geotaxis mature or first appear on this day in the mouse ${ }^{[1]}$ and because of continued neuronal and glial development in other brain regions ${ }^{[15,19,20]}$. Of importance, VPA administration results in high levels of markers for oxidative stress and lipid peroxidation including 15-F-isoprostane and thiobarbituric acid reactive substances ${ }^{[21-23]}$.

An organic mercury, $\mathrm{MeHg}$, was selected as our second compound for testing because it is an important, widely distributed environmental toxicant. $\mathrm{MeHg}$ does cross the placental barrier and, in humans exposed in utero to acute high doses, was shown to cause retardation in cognitive and locomotor development along with numerous other neurological symptoms including seizures and cerebral palsy ${ }^{[24]}$. Nonetheless, it is important to note that autism was not found to be associated with either pre- or neonatal exposure to organic mercury.

The consequences of low dose, chronic exposure to mercury through fish consumption are somewhat more controversial with some studies showing deleterious effects while others show no adverse consequences ${ }^{[2,24]}$. Early exposure to mercury has been shown to disrupt the neurobehavioral development of other species including rodents and primates ${ }^{[25]}$. The mechanism through which $\mathrm{MeHg}$ exerts its toxicity is thought to be, in part, mediated by disruption of neural cell adhesion molecules ${ }^{[26]}$. In addition, oxidative stress is involved in $\mathrm{MeHg}$-induced neurotoxicity as demonstrated by increased ROS and thiobarbituric acid reactive substances and a reduction in GSH levels ${ }^{[27]}$. In addition, the neurotoxicity of $\mathrm{MeHg}$ in cultured neurons was blocked by the pretreatment with antioxidants ${ }^{[28]}$. Trolox, a water-soluble derivative of vitamin E, protects against MeHg-induced neurotoxicity in rats ${ }^{[29]}$. Likewise, antioxidants produced protective effects against $\mathrm{MeHg}$ toxicity in cultured human neurons and astrocytes $^{[30]}$. Indeed, ROS have been implicated in MeHg-induced neurotoxicity in multiple experimental models ${ }^{[27,31-34]}$. Finally, we have recently demonstrated that pretreatment with Trolox protects mice against the neurobehavioral deficits induced by postnatal $\mathrm{MeHg}^{[8]}$. Collectively, these data indicate that early exposure to $\mathrm{MeHg}$ causes neurobehavioral deficits consequent, at least in part, to the generation of ROS.

In summary, wide ranges of toxicants and genetic alterations have been associated with autism. The toxicants are thought to have a common mechanism of generating $\operatorname{ROS}^{[4-6]}$ while the genetic alterations are thought to result in enhanced sensitivity to the deleterious effects of ROS. Accordingly, we now hypothesize that autism may be the result multiple exposures to any of a number of toxicants; the initial exposure sensitizes the subject such that later exposures to the same or different toxicants results in an enhanced 
oxidative stress response. Furthermore, we predict that this sensitization will be exacerbated in individuals with genetic alterations affecting their handling of ROS. In previous studies we have demonstrated a sensitization response to dopaminergic toxicants in adult mice following prenatal administration of $\mathrm{MeHg}^{[7]}$. We have also demonstrated that antioxidant pretreatment protects mice against the behavioral deficits induced by early exposure to $\mathrm{MeHg}^{[8]}$. Accordingly, the objective of this study was to determine if antioxidants administered as a pretreatment to VPA would protect the mice against the VPA-induced behavioral regression. In addition, we sought to determine if the early VPA administration would alter levels of glial fibrillary acidic protein (GFAP), a marker of astrocytic neural injury, thus serving as a biological marker for the VPA-induced behavioral deficits.

\section{MATERIALS AND METHODS}

Subjects: Male and female BALB/c mice (Taconic, Germantown, NY) were housed together in plastic cages with standard wood chip bedding and free access to food and water. All mice were maintained in an AAALAC-accredited facility under guidelines set forth by the National Institutes of Health. Lights were set on a $12 \mathrm{~h}$ on: $12 \mathrm{~h}$ off cycle and temperature was maintained at $25^{\circ} \mathrm{C}$. Females were checked before 10 AM for presence of a vaginal plug which was recorded as day 0 of embryonic development. Day of birth was recorded as day 0 and all pups were labeled for individual identification. Body weight was measured daily. Female pups were removed from the cage on day 5. For the behavioral studies, the sodium valproate (Sigma) dose was $400 \mathrm{mg} \mathrm{kg}^{-1}$ with a saline vehicle and the vitamin $\mathrm{E}$ dose was also $400 \mathrm{mg} \mathrm{kg}^{-1}$ but with a corn oil vehicle. All injections were s.c. in a volume of $1.0 \mathrm{~mL} 100^{-1} \mathrm{~g}$ body weight.

Negative geotaxis: Negative geotropism was tested on postnatal days $\mathrm{P} 13-19$ by placing the mouse facing downward along a $45^{\circ} \mathrm{C}$ incline. Latency to turn $180^{\circ} \mathrm{C}$ such that the head was facing upward along the incline was recorded with a maximum of 30 seconds for each trial.

Motor Activity: Motor activity was assessed on days P14-19. The chamber consisted of a black $42 \times 22 \times 14 \mathrm{~cm}$ Plexiglass box. Six infrared sensors placed approximately $7 \mathrm{~cm}$ apart and $2.5 \mathrm{~cm}$ above the floor were used to measure activity over a $10 \mathrm{~min}$ period.
Mid-air righting: When a mouse is dropped upside down from a height of $45 \mathrm{~cm}$ onto a padded surface it engages in a mid-air righting reflex with orderly, rostrocaudal movements, initiated with head and concluded with the hindlimbs such that the animal lands on its paws. The behavior first appears on P13 and is fully achieved by $\mathrm{P} 17^{[35]}$. Mid-air righting has been linked to cerebellum development ${ }^{[36]}$. For the mid-air righting test, mice were elevated $45 \mathrm{~cm}$ above a foam pad, dorsal side down. The animal was released and ability to right in mid air assessed scored as the mouse landing on its paws on two out of three trials each day. Mice were tested on P13-20.

Protein determination: In order to determine changes in protein expression following VPA treatment at behaviorally significant time points, animals were treated with VPA $600 \mathrm{mg} \mathrm{kg}^{-1}$ or saline on $\mathrm{E} 13^{[1]}$ and assayed on days P4 and P5 with the cerebellum removed and stored at $-70^{\circ} \mathrm{C}$. Protein analysis via gel electrophoresis, western blot and densiometry was performed according to the methods of Dey et al. ${ }^{[26]}$ with some small variations. In summary, whole homogenate fractions were homogenized in 1:10 w/v of a Tris extraction buffer $[50 \mathrm{mM}$ TrisHCl, $\mathrm{pH} 7.4$, $0.32 \mathrm{M}$ sucrose, $1 \mathrm{mM}$ EDTA, 1 vial to $100 \mathrm{ml}$ protease inhibitor (Sigma, St. Louis, MO). The supernatant was removed following centrifugation for $10 \mathrm{~min}$ at $1000 \mathrm{xG}$ and combined with equilibration buffer $[0.125 \mathrm{M}$ TrisHCl, $\mathrm{pH}$ 6.8, 4\% SDS, 20\% glycerol, 10\% mercapoethanol) and immediately heated for $30 \mathrm{~min}$ at $70^{\circ} \mathrm{C}$. Protein values were determined using the BCA protein assay (Pierce, Rockford, IL) modified for a BOBAS FARA II enzyme analyzer (Roche Diagnostics, Nutley, NJ). Samples of $10 \mu \mathrm{g}$ protein were separated by SDS-PAGE on a $10 \%$ polyacrylamide gradient gel using a Bio-Rad MiniProtean II System (Bio-Rad, Mellville, NY) for GFAP and synaptophysin. Proteins were transferred to nitrocellulose membranes and were washed twice for 10 min each in phosphate buffered saline (PBS) and blocked with 5\% non-fat dry milk in PBS for $1 \mathrm{~h}$ prior to application of primary antibody. Immunoblotting for GFAP and synaptophysin was performed overnight at $4^{\circ} \mathrm{C}$. All primary antibodies were obtained from Fisher (Springfield, NJ). Anitgens were visualized following $1 \mathrm{~h}$ incubation with secondary peroxidase antibodies (Southern Biotechnology Associates, Birmingham, AL) and application of chemiluminescence ECL substrate detection on Hyperfilm ECL autoradiographic film (Amersham). For GFAP detection, this method was verified in a separate study using a dose response of trimethyltin treatment using a GFAP protein standard 
(Chemicon, Inc.). ECL images were scanned into an IBM PC using a Hewlett Packard Scanner with a transparency adapter. Densiometric analysis was performed using Image Pro Analysis Software using percent of saline treated controls as the standard.

Statistical analysis: All behavioral analysis were performed using a repeated measures ANOVA including both group, day and sex as main factors, with the exception of the mid-air righting response which was analyzed using Chi-Square and Fisher's Exact Test.

\section{RESULTS AND DISCUSSION}

Negative geotaxis: Control mice and those treated with vitamin $\mathrm{E}$ alone were able to perform the reflexive negative geotaxis response, reorienting their head to point upward when placed on an inclined plane with their head facing down. This reflex improved across development, as the latency to re-orient improved across testing $(\mathrm{F}(6,312)=5.4, \mathrm{p}<0.001)$. VPA-treated mice displayed an increased latency to perform this reorientation response $(\mathrm{F}(1,52)=10.0, \mathrm{p}<0.005)$. Posthoc analysis revealed that following day 14 treatment with VPA, there was a significant regression in the performance of this response, which reached statistical significance on days 16 and 17. Importantly, this VPAinduced regression was blocked by vitamin $\mathrm{E}$ pretreatment, such that pretreated mice were able to perform this response with a similar latency as controls on P16 and P17 $(\mathrm{F}(1,52)=5.3, \mathrm{p}<0.05)$. Finally, VPAtreated mice regained their ability to perform this behavioral response similar to controls by the completion of testing on P19 (Fig. 1).

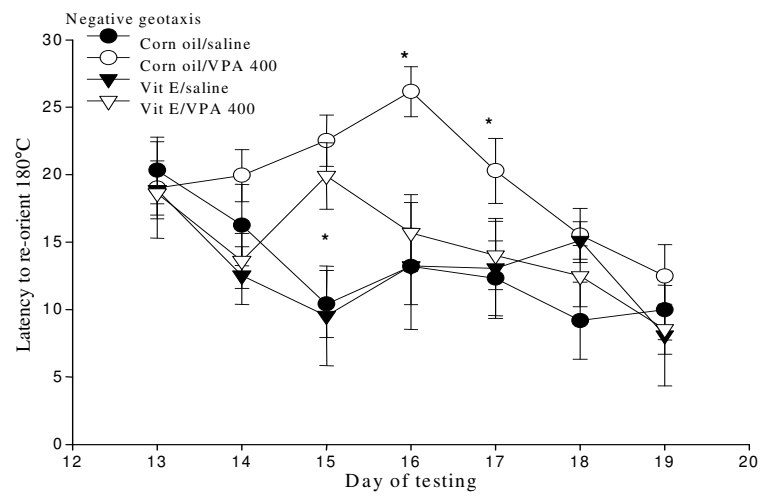

Fig. 1: Negative geotaxis: Latency to reorient from head down to head up on a $45^{\circ} \mathrm{C}$ incline for groups of pups treated with VPA (400 mg kg-1) or saline on P14. Some groups received vitamin E pretreatment while others received corn oil. *: $\mathrm{p}<0.05$ compared to corn oil/saline

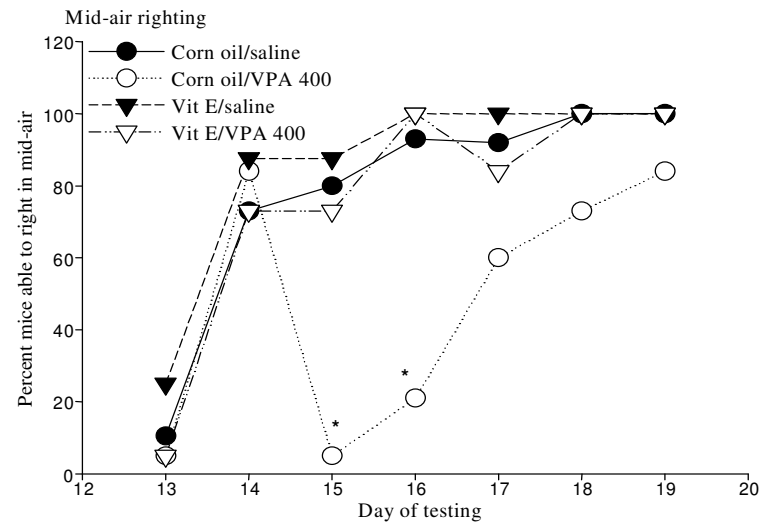

Fig. 2: Mid-air righting: Number of pups successfully engaging in mid-air righting (expressed as a percent of pups mid-air righting on 2 out of 3 trials/day) for groups of pups treated with VPA $\left(400 \mathrm{mg} \mathrm{kg}^{-1}\right)$ or saline on P14. Some groups received vitamin $\mathrm{E}$ pretreatment while others received corn oil. *: $\mathrm{p}<0.05$ compared to corn oil/saline

Mid-air righting: Before any treatment was administered, less than $20 \%$ of the pups were able to engage in mid-air writing on P13 but this improved to about $75 \%$ by $\mathrm{P} 14$. This observation is interpreted to indicate that cerebellar and general muscular maturation have matured by P14. $\chi^{2}$ analysis revealed that following VPA-treatment given after behavioral testing on P14 caused a regression in mid-air righting on P15 $\left[\chi^{2}(3)=39.8, \mathrm{p}<0.0001\right]$ when compared to saline controls. This regression was still observed on P16 in the VPA-treated animals. However, the VPA-induced regression was eliminated by pretreatment with vitamin E (Fig. 2).

Motor activity: Mice engaged in a similar amount of locomotor activity at the start of testing. This activity significantly increased across post-natal development $[\mathrm{F}(1,38)=80.2, \mathrm{p}<0.001]$. Interestingly, there was a trend for mice treated with VPA to engage in intrusive behaviors following P14 treatment. This was evidenced by increased levels of activity across testing, beginning on P16 through P18. However, this hyperactivity did not reach statistical significance. In addition, this hyperactive behavior was blocked by pre-treatment with vitamin E (Fig. 3).

GFAP and synaptophysin: Previous work in our lab revealed retarded neurobehavioral development in mice treated with $600 \mathrm{mg} \mathrm{kg}^{-1}$ VPA on embryonic day $13^{[1]}$. In order to determine whether we could detect a 


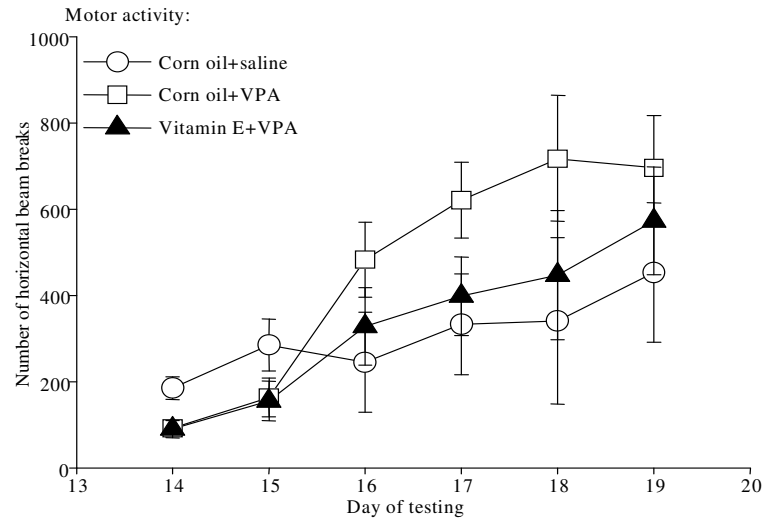

Fig. 3: Motor activity: Horizontal beam breaks for groups of pups treated with VPA (400 mg kg ${ }^{-1}$ ) or saline on P14. Some groups received vitamin E pretreatment while others received corn oil. $*$ : $\mathrm{p}<0.05$ compared to corn oil/saline
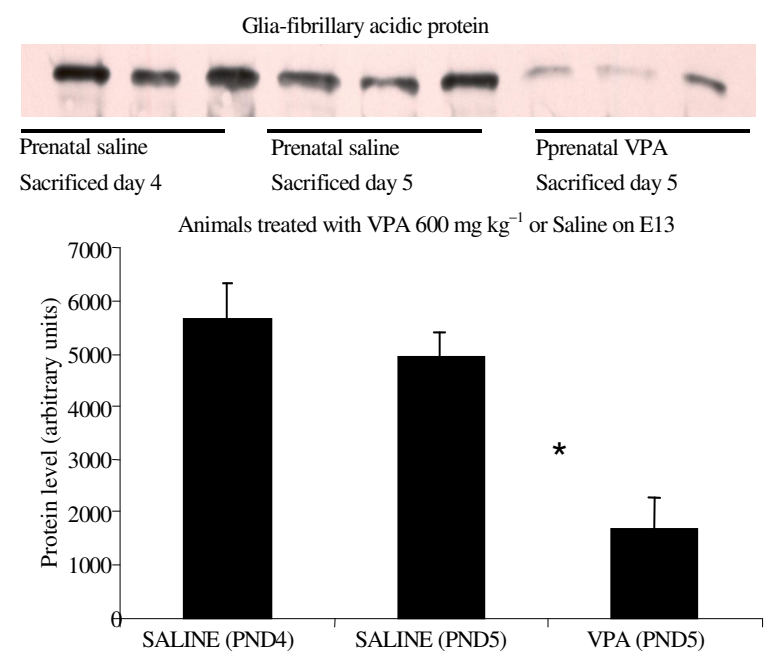

Fig. 4: GFAP: Pups received either VPA $\left(600 \mathrm{mg} \mathrm{kg}^{-1}\right)$ or saline in utero on E13 and were sacrificed on either P4 or P5. *: $\mathrm{p}<0.05$ compared to corn oil/saline

biological marker of VPA-induced neurobehavioral retardation, we examined early postnatal levels of GFAP and synaptophysin in the cerebellum following in utero exposure to VPA. Mice treated with $600 \mathrm{mg} \mathrm{kg}^{-1}$ VPA on E13 and sacrificed on P5 showed decreased concentrations of GFAP in the cerebellum compared to saline treated controls sacrificed on both P4 and P5 (F $(2,8)=12.3, \mathrm{p}<0.01)$ (Fig. 4). Likewise, the concentration of synaptophysin was significantly decreased in the cerebellum of E13 VPA-treated mice compared to both P4 and P5 saline controls

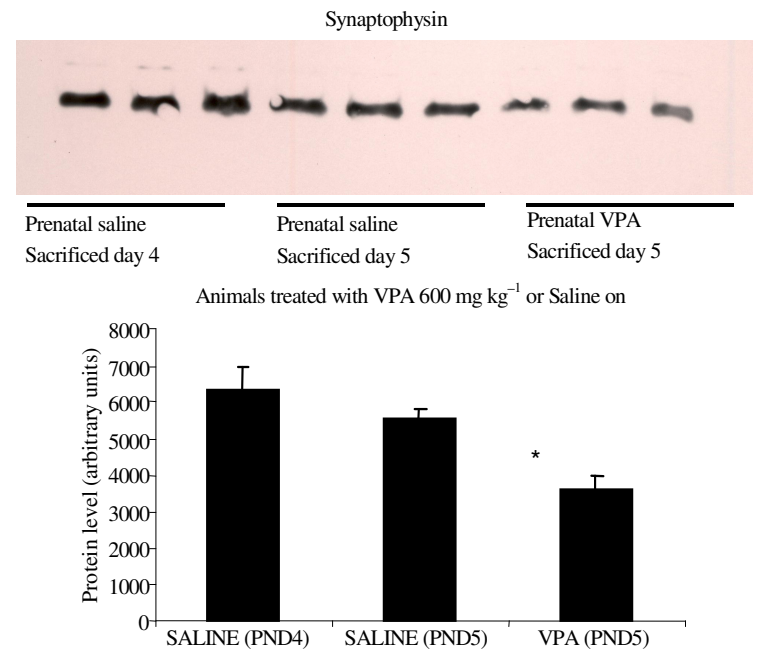

Fig. 5: Synaptophysin: Pups received either VPA $\left(600 \mathrm{mg} \mathrm{kg}^{-1}\right)$ or saline in utero on E13 and were sacrificed on either P4 or P5. *: p<0.05 compared to corn oil/saline

$(\mathrm{F}(2,6)=10.0, \mathrm{p}<0.01)$ (Fig. 5). During this period of postnatal development, GFAP and synaptophysin levels increase to promote normal astrocytes-neuron interactions and synaptogensis, respectively ${ }^{[37]}$. Therefore, mice treated with VPA in utero show immature neural development, since the levels of GFAP and synaptophysin observed on P5 are much lower than those found in mice from an earlier postnatal period (P4). This suggests that the behavioral retardations seen in our previous study are influenced by retarded neural development.

The etiology of autism is thought to involve early exposure to ROS-generating toxicants acting upon genetically-sensitive individuals. We have developed a new strategy to assess the detrimental effects of early toxicant exposure on neurobehavioral development, classifying the behavioral deficits as retardations, regressions or intrusions. In previous studies we demonstrated that early exposure to VPA or $\mathrm{MeHg}$ results in behavioral deficits in the maturation of social, cognitive and motor skills ${ }^{[1,8]}$. Furthermore, we demonstrated that our behavioral model was useful in demonstrating that pretreatment with an antioxidant protected mice against the behavioral deficits induced by early exposure to $\mathrm{MeHg}^{[8]}$. In the present study, we demonstrated that vitamin $\mathrm{E}$ was capable of protecting mice against VPA-induced regression in negative geotaxis and mid-air righting as well as against intrusive VPA-induced hyperactivity. Collectively, the present data together with our previous $\mathrm{MeHg}$ study indicate that the generation of ROS may be a common 
factor mediating toxicant-induced neuronal damage associated with autism and that neurobehavioral assessments provide an important functional measure of the potential benefits of antioxidants.

A second objective of the present study was to develop a biological marker of the VPA-induced damage. Toward this end we used our initial model, delivering the VPA prenatally on E13 ${ }^{[1]}$. We had demonstrated that this prenatal VPA treatment resulted in later behavioral deficits as assessed in the surface and mid-air righting tests, negative geotaxis and in water maze. Furthermore, this prenatal VPA treatment resulted in sex-dependent differences in these behavioral deficits with males more affected than females. In the present study, we found that both cerebellar GFAP and cerebellar synaptophysin were reduced postnatally following the prenatal VPA administration. GFAP is a marker of astroglia in the brain and is involved in astrocyte-neuron interactions. GFAP mutant mice have abnormal structure and exhibit deficient long-term depression in cerebellar Purkinje cell synapses ${ }^{[37]}$. Therefore, major alterations in GFAP may alter Purkinje cell communication that, in turn, may alter behavior. Synaptophysin is a widely used marker for nerve terminals and can indicate synaptogenesis. Therefore, a reduction in synaptophysin in the cerebellum could signify a reduction in synatpogenesis in that region. More generally, it is intriguing that these biological markers may reflect the behavioral deficits of cognitive and motor retardation caused by the early VPA exposure. Future studies are designed to determine if the antioxidant pretreatment also protects the mice against these neurological changes induced by the VPA.

There is ample evidence that ROS are involved in human autism. Free oxygen radicals could result from ingested or inhaled environmental toxins, food or food additives, inflammation or infection (overt or occult). The interaction of free oxygen radicals and polymorphic oxidative genes during gestation or postnatally could disrupt neurogenesis in developing brain at multiple time windows, eliciting immediate stage-dependent effects in specific systems that influence subsequent ontogenetic processes, leading to the phenotype of autism. Indeed an exacerbated oxidative stress response has been implicated in autism. Specifically lower glutathione peroxidase (GPX) and superoxide dismutase (SOD) activity were found in children with autism ${ }^{[38-40]}$. An increase in body burden of various toxins was reported in autism ${ }^{[41,42]}$. In addition, provoked urinary mercury excretion is found to be higher in autism ${ }^{[43]}$. These toxins could generate oxidative stress in children with autism. Elevated nitrite and nitrate in plasma ${ }^{[44]}$ and red cells ${ }^{[38]}$ have been reported in children with autism. This elevation indicates excess generation of nitric oxide free radicals. In addition, two independent double blind placebo controlled clinical trials of antioxidants (vitamin $\mathrm{C}$ or carnosine) showed beneficial effects in autism ${ }^{[45,46]}$. Finally, we conducted a study of oxidative stress biomarkers in children with autism and age matched healthy controls. Our results showed that urinary excretion of 8 isoprostane $F_{2 \alpha}$ was significantly higher in children with autism as compared to healthy controls $^{[47]}$. There was also a trend of increased 8OHdG urinary excretion in autistic subjects. These results suggest that oxidative stress is exacerbated in autism and are consistent with the present results of antioxidant protective effects against VPA-induced behavioral deficits in mice.

In summary, we have developed a comprehensive neurobehavioral model in which mice are exposed to candidate toxicants during critical periods of neural development. The mice may have altered expression of genes thought to be associated with autism and/or to confer increased sensitivity to the toxicants. The mice are then assessed in a battery of tests designed to assess behavioral maturation of skills in the social, cognitive and motor domains. Toxicant or genetic-induced deficits in the behavioral maturation are classified as retardations, regressions or intrusions. In the present studies, we further demonstrate that pretreatment with an antioxidant protects the mice against the toxicantinduced behavioral deficits. We conclude that our model is useful for evaluation of the theory that oxidative stress may play a role in the etiology of autism.

\section{ACKNOWLEDGEMENT}

Supported by ES11256, EPAR829391, New Jersey Governor's Council on Autism and Autism Speaks.

\section{REFERENCES}

1. Wagner, G.C., K.R. Reuhl, M. Cheh, M., P. McRae and A.K. Halladay, 2006. A new neurobehavioral model of autism in mice: Pre- and Postnatal exposure to sodium valproate. J. Autism Dev. Dis., 36: 779-793.

2. Bernard, S., A. Enayati, L. Redwood, H. Roger and T. Binstock, 2000. Autism: A novel form of mercury poisoning. ARC Res., 1-12.

3. Dawson, G., 1996. Neuropsychology of autism: a report on the state of the science. J. Aut. Dev. Dis., 26: 179. 
4. Chauhan, A. and V. Chauhan, 2006. Oxidative stress in autism. Pathophysiol., 13: 171-181.

5. Chauhan, A., V. Chauhan, T. Brown and I. Cohen, 2004. Oxidative stress in autism: increased lipid peroxidation and reduced serum levels of ceruloplasmin and transferin-the antioxidant proteins. Life Sci., 75: 2539-2549.

6. James, S.J., P. Cutler, S. Melnyk, S. Jernigan, L. Janak, D.W. Gaylor and J.A. Neubrander, 2004. Metabolic biomarkers of increased oxidative stress and impaired methylation capacity in children with autism. Am. J. Clin. Nutr., 80: 1611-1617.

7. Wagner, G.C., K.R. Reuhl, X. Ming and A.K. Halladay, 2007. Behavioral and neurochemical sensitization to amphetamine following early postnatal administration of methylmercury. NeuroToxicol., 281: 59-66.

8. Cheh, M.A., A.K. Halladay, K.R. Reuhl, M. Polunas, X. Ming and G.C. Wagner, 2007. Trolox, a Vitamin $\mathrm{E}$ derivative, protects against persistent neurobehavioral disruption induced by neonatal methylmercury exposure. Soc. Toxicol., NC.

9. Rodier, P.M., J.L. Ingram, B. Tisdale, S. Nelson and J. Romano, 1996. Embryological origin for autism: developmental abnormalities of the cranial nerve motor nuclei. J. Comp. Neurol., 370:247-261.

10. Ingram, J.L., S.M. Peckham, B. Tisdale and P.M. Rodier, 2000a. Prenatal exposure to valproic acid reproduces the cerebellar anomalies associated with autism. Neurotox. Teratol., 22, 319-324.

11. Sobaniec-Lotoweska, M.E., 2001. Ultrastructure of purkinje cell perikara and their dendritic processes in the rat cerebellar cortex in experimental encephalopathy induced by chronic application of valproate. Int. J. Exp. Pathol., 82: 337-348.

12. Moore, S.J., P. Turnpenny, A. Quinn, S. Glover and D.J. Lloyd, 2000. Montgomery and J.C.S. Dean, A clinical study of 57 children with fetal anticonvulsant syndromes. J. Med. Genetics, 37: 489-497.

13. Williams, G., J. King, M. Cunningham, M. Stephan, B. Kerr and J.H. Hersh, 2001. Fetal valproate syndrome and autism: additional evidence of an association. Dev. Med. Child Neurol., 43: 202-206.

14. Chapman, J.B. and M.G. Cutler, 1989. Effects of sodium valproate on development and social behaviour in the Mongolian gerbil. Neurotoxicol. Teratol., 11: 193-198.
15. Voorhees, C.V., 1987. Behavioral teratogenicity of valproic acid: selective effects on behavior after prenatal exposure to rats. Psychopharmacol., 92: 173-179.

16. Schneider, T. and R. Przewlocki, 2005. Behavioral alterations in rats prenatally exposed to valproci acid: Animal model of autism. Neuropsychopharmacol., 30: 80-89.

17. Altman, J. and S.A. Bayer, 1978. Prenatal development of the cerebellar system in the rat. Cytogenesis and histogenesis of the deep nuclei and cortex of the cerebellum. J. Comp. Neurol., 179: 23-48.

18. Inouye, M. and U. Murakami, 1980. Temporal and spatial patterns of purkinje cell formation in the mouse cerebellum. J. Comp. Neurol., 194: 499-503.

19. Bachevalier, J. and M. Beauregard, 1993. Maturation of medial temporal lobe memory functions in rodents, monkeys and humans. Hippocampus, 3:191-202.

20. Rice, D. and S. Barone, 2000. Critical periods of vulnerability for the developing nervous system: Evidence from human and animal models. Environ. Health Per., 108: 511-533.

21. Murugesan, V. and P. Subramanian, 2003. Enhancement of circulatory antioxidants by $\alpha$ ketoglutarate during sodium valproate treatment in wistar rats. Polish J. Pharmacol., 55: 31-36.

22. Tong, V., T.K.H. Chang, J. Chen and F.S. Abbott, 2003. The effect of valproic acid on hepatic and plasma levels of 15-F2t-Isoprostane in rats. Free Radical Biol. Med. 34: 1435-1446.

23. Sobaniec-Lotowska, M.E., 1997. Effects of longterm administration of the antiepileptic drugsodium valproate upon the ultrastructure of hepatocytes in rats. Exp. Toxicol. Pathol., 49: 225-32.

24. Myers, G.J. and P.W. Davidson, 2000. Does methylmercury have a role in causing developmental disabilities in children? Environ. Health Per., 108: 413-420.

25. Rice, D.C., 1996. Sensory and cognitive effects of developmental methylmercury exposure in monkeys and a comparison to effects in rodents. Neurotoxicol., 17, 139-154.

26. Dey, P.M. and K.R. Reuhl, 1999. Developmental methylmercury administration alters cerebellar PSA-NCAM expression and Golgi sialyltransferase activity. Brian Res., 845:139-151.

27. Yee, S. and B.H. Choi, 1996. Oxidative stress in neurotoxic effects of methylmercury poisoning. Neurotoxicol., 17: 17. 
28. Park, S.T., K.T. Lim, Y.T. Chung and S.U. Kim, 1996. Methylmercury-induced neurotoxicity in cerebral neuron culture is blocked by antioxidants and NMDA receptor antagonists. Neurotoxicol., 17: 37 .

29. Usuki, F., A. Yasutake, F. Umehara, H. Tokunaga and M. Matsumoto et al., 2001. In vivo protection of a water-soluble derivative of vitamin E, Trolox, against methylmercury-intoxication in the rat Neurosci Lett., 304: 199-203. 30. Sanfeliu, C., J. Sebastia and S.U. Ki, 2001. Methylmercury neurotoxicity in cultures of human neurons, astrocytes, neuroblastoma cells. Neurotoxicol., 22: 317-327.

31. Aschner, M., C.P. Yao, J.W. Allen and K.H. Tan, 2000. Methylmercury alters glutamate transport in astrocytes. Neurochem. Int., 37: 19.

32. Stohs, S.J. and D. Bagchi, 1995. Oxidative mechanisms in the toxicity of metal ions. Free Radical Biol. Med., 18: 321.

33. Sorg, O., B. Schilter, P. Honegger and F. Monnet-Tschudi, 1998. Increased vulnerability of neurones and glial cells to low concentrations of methylmercury in a prooxidant situation. Acta Neuropathol. (Berl), 96: 621.

34. Mundy, W.R. and T.M. Freudenrich, 2000. Sensitivity of immature neurons in culture to metal-induced changes in reactive oxygen species and intracellular free calcium. Neurotoxicol, 21: 1135 .

35. Altman, J. and K. Sudarshan, 1975. Postnatal development of locomotion in the laboratory rat. Animal Behav., 23: 896-901.

36. Petrosini, L., M. Molinari and T. Gremoli, 1990. Hermicerebellectomy and motor behavior in rats. II. Effects of cerebellar lesion performed at different developmental stages. Exp. Brain Res., 82: 483-492.

37. Shibuki, K., H. Gomi, L. Chen, S. Bao, J.J. Kim, H. Wakatsuki, T. Fujisaki, K. Fujimoto, A. Katoh, T. Ikeda, C. Chen, R.F. Thompson and S. Itohara, 1996. Deficient cerebellar Long-term depression, impaired eyeblink conditioning and normal motor coordination in GFAP mutant mice. Neuron, 16: 587-599.
38. Sogut, S., S.S. Zoroglu and H. Ozyurt et al., 2003. Changes in nitric oxide levels and antioxidant enzyme activities may have a role in the pathophysiological mechanisms involved in autism. Clinica Chimica Acta, 331: 111-117.

39. Golse, B., P. Debray-Ritzen and P. Durosay et al., 1978. Perturbation de deux enzymes, la superoxyde-dismutase I et la glutathioneperoxydase dans la psychose infantile de developpement (autisme infantile). Rev. Neurol. (Paris), 134: 699-705.

40. Yorbik, O., A. Sayal and C. Akay et al., 2002. Investigation of antioxidant enzymes in children with autistic disorder. Prostaglandins Leukot Essent Fatty Acids, 67: 341-343.

41. Edelson, S.B. and D.S. Cantor, 1998. Autism: xenobiotic influences. Toxicol. Ind. Health, 14: 799-811.

42. Edelson S.B. and D.S. Cantor, 2000. The neurotoxic etiology of the autistic spectrum disorder: a replicative study. Toxicol. Ind. Health, 16: 239-47.

43. Bradstreet, J., D.A. Geier and J.J. Kartzinel et al., 2003. A case-control study of mercury burden in children with autistic spectrum disorders. J. Am. Phy. Sur., 8: 76-79.

44. Zoroglu, S.S., M. Yurekli and I. Meram et al., 2003. Pathophysiological role of nitric oxide and adrenomedullin in autism. Cell Biochem. Function, 21: 55-60.

45. Chez, M.D., C.P. Buchanan and M.C. Aimonovitch et al., 2002. Double-blind, placebo-controlled study of 1-carnosine supplementation in children with autistic spectrum disorders. J. Child Neurol., 17: 833-837.

46. Dolske, M.C., J. Spollen and S. McKay et al., 1993. A preliminary trial of ascorbic acid as supplemental therapy for autism. Prog. NeuroPsychopharm. Biol. Psychiat., 17: 765-774.

47. Ming, X., P. Stein, M. Brimacombe, W. Johnson, G. Lambert and G.C. Wagner, 2005. Increased excretion of a lipid peroxidation biomarker in autism. Prostaglandins, Leukotrienes and Essential Fatty Acids, 73: 379-384. 